\title{
GENERAL RELATIVISTIC EFFECTS ON NONLINEAR POWER SPECTRA
}

\author{
Donghui JeOng ${ }^{1}$, Jinn-Ouk Gong ${ }^{2,5}$, Hyerim NOH $^{3}$, AND JAI-CHAN HWAng ${ }^{4}$ \\ ${ }^{1}$ California Institute of Technology, Pasadena, CA 91125-1700, USA; djeong@tapir.caltech.edu \\ 2 Instituut-Lorentz for Theoretical Physics, Universiteit Leiden, 2333 CA Leiden, The Netherlands; jgong@lorentz.leidenuniv.nl \\ ${ }^{3}$ Korea Astronomy and Space Science Institute, Daejeon 305-348, Republic of Korea; hr@kasi.re.kr \\ ${ }^{4}$ Department of Astronomy and Atmospheric Sciences, Kyungpook National University, Daegu 702-701, Republic of Korea; jchan@knu.ac.kr \\ Received 2010 November 11; accepted 2010 November 12; published 2010 December 28
}

\begin{abstract}
The nonlinear nature of Einstein's equation introduces genuine relativistic higher order corrections to the usual Newtonian fluid equations describing the evolution of cosmological perturbations. We study the effect of such novel nonlinearities on the next-to-leading order matter and velocity power spectra for the case of a pressureless, irrotational fluid in a flat Friedmann background. We find that pure general relativistic corrections are negligibly small over all scales. Our result guarantees that, in the current paradigm of standard cosmology, one can safely use Newtonian cosmology even in nonlinear regimes.
\end{abstract}

Key words: cosmology: theory - large-scale structure of universe

Online-only material: color figures

\section{INTRODUCTION}

The large-scale structure (LSS) of the universe is a powerful probe for studying the nature of cosmological density perturbations and for extracting cosmological parameters (Peebles 1980). Combined with the anisotropy of the cosmic microwave background (CMB), most of the cosmological parameters are currently constrained to within a few percent accuracy or even better (Komatsu et al. 2010). To continue our success in cosmology with CMB and LSS, it is crucial to predict the power spectra from theory accurately. While the temperature fluctuations in the CMB is as small as $\delta T / T \sim 10^{-5}$ (Smoot et al. 1992) so that linear perturbation theory is able to provide the necessary accuracy, we have a larger degree of nonlinearities in LSS. We must properly take into account nonlinearities of LSS to predict the power spectrum accurately enough for precision cosmology at a level similar to the CMB (Jeong \& Komatsu 2006, 2009).

Most studies on LSS, however, have been based on Newtonian gravity, especially those including nonlinear perturbations (Vishniac 1983; Goroff et al. 1986; Makino et al. 1992; Fry 1994; Bernardeau et al. 2002). This approach has to be justified a posteriori by comparing the results with those based on the fully general relativistic treatment. For example, in Noh \& Hwang (2004), it is shown that the Newtonian hydrodynamic equations up to second order coincide exactly with the relativistic ones in the zero-pressure case, after appropriately identifying hydrodynamical variables with gauge-invariant combinations of relativistic perturbation variables. Thus, compared to the Newtonian hydrodynamic equations which are closed at second order, any higher order contributions originate from purely general relativistic effects (Hwang \& Noh 2005b). A consistent expansion of the density fluctuation tells us that the leading nonlinear contributions to the power spectrum include third-order perturbations (Noh \& Hwang 2008; Noh et al. 2009). Thus, nonlinear density power spectrum naturally includes pure general relativistic effects, which may have important implications as

\footnotetext{
5 Current address: Theory Division, CERN, CH-1211 Genève 23, Switzerland.
}

$\mathrm{N}$-body simulations are becoming larger and larger in order to reach the horizon scale (Kim et al. 2009).

In this paper, we examine the general relativistic effects on the power spectra of matter density fluctuations and peculiar velocity by including leading non-vanishing, nonlinear contributions. Our aim is to answer the question whether pure general relativistic effects can give rise to any cosmologically observable consequences. To our surprise, we find that the Newtonian terms in these power spectra are absolutely dominating over all relevant cosmological scales, even outside the horizon. Although the result sounds simple and pleasant, this is still a non-trivial result, because in the context of cosmology, Newtonian gravity is incomplete: there is no concept of horizon, the propagation speed of an action at one point is infinite, and so on.

This paper is outlined as follows. In Section 2, we present the formalism to set up the equations to solve, and give the solutions up to third order. In Section 3, we compute the matter and velocity power spectra including next-to-leading nonlinear corrections which include genuine general relativistic effects. In Section 4, we conclude.

\section{EQUATIONS AND SOLUTIONS}

We consider the Einstein-de Sitter universe, i.e., a flat universe dominated by pressureless, irrotational matter, and consider only the scalar perturbations. We work in the temporal comoving gauge where $T^{0}{ }_{i}=0$ to all perturbation orders, with $i$ being a spatial index. As this temporal gauge condition, together with our unique spatial gauge condition $g_{i j}=a^{2}(1+2 \varphi) \delta_{i j}$ (Bardeen 1988), fixes the gauge degrees of freedom completely, all the resulting perturbation variables can be equivalently regarded as fully gauge invariant, both spatially and temporally. This statement is valid in all perturbation orders (Noh \& Hwang 2004).

The Arnowitt-Deser-Misner formulation (Arnowitt et al. 2008) is convenient in our case (Bardeen 1980). In the comoving gauge, we set the momentum density to be zero, i.e., $J_{i} \equiv$ $N T^{0}{ }_{i}=0$, with $N$ being the lapse function. The pressureless condition implies $S_{i j} \equiv T_{i j}=0$. Therefore, the momentum conservation equation gives $N_{, i}=0$; thus, the lapse function $N$ 
is uniform. The energy conservation and momentum constraint equations and the trace part of the propagation equation then become (Bardeen 1980)

$$
\begin{gathered}
E_{, 0}-N^{i} E_{, i}=N K E, \\
\bar{K}^{j}{ }_{i \mid j}-\frac{2}{3} K_{\mid i}=0, \\
K_{, 0}-N^{i} K_{, i}=N\left(\frac{1}{3} K^{2}+\bar{K}^{i}{ }_{j} \bar{K}^{j}{ }_{i}+4 \pi G E-\Lambda\right),
\end{gathered}
$$

where $N_{i}$ is the shift vector, $K \equiv K^{i}{ }_{i}$ is the trace of the extrinsic curvature tensor $K_{i j}, \Lambda$ is the cosmological constant, and $E \equiv N^{2} T^{00}$ is the energy density, and an overbar denotes the traceless part, while a vertical bar denotes a covariant derivative with respect to $g_{i j}$. These are the complete equations we need in our nonlinear perturbations, and they are valid in fully nonlinear situations. Note that in the above equations, we set $c=1$.

We introduce the density and the velocity fluctuations as $E \equiv \rho(t)+\delta \rho(t, \boldsymbol{x})$ and $K \equiv 3 H-\theta(t, \boldsymbol{x})$ with $\theta(t, \boldsymbol{x}) \equiv a^{-1} \nabla$. $\boldsymbol{u}(t, \boldsymbol{x})$, with $a$ being the cosmic scale factor. We can identify $\delta \rho(t, \boldsymbol{x})$ and $\boldsymbol{u}(t, \boldsymbol{x})$ as the Newtonian density and velocity perturbation variables, respectively, because the relativistic equations coincide exactly with the corresponding Newtonian hydrodynamic equations up to second order.

From the above equations, we can derive the hydrodynamic equations of density fluctuation $\delta(t, \boldsymbol{x}) \equiv \delta \rho(t, \boldsymbol{x}) / \rho(t)$ and velocity divergence $\theta(t, \boldsymbol{x})$ to the third order (Hwang \& Noh $2005 b)$. The relativistic continuity and Euler equations are found to be

$$
\frac{\partial \delta}{\partial t}+\frac{1}{a} \nabla \cdot \boldsymbol{u}=-\frac{1}{a} \nabla \cdot(\delta \boldsymbol{u})+\frac{1}{a}\left[2 \varphi \boldsymbol{u}-\nabla\left(\Delta^{-1} X_{2}\right)\right] \cdot(\nabla \delta),
$$

$$
\begin{aligned}
& \frac{1}{a} \nabla \cdot\left(\frac{\partial \boldsymbol{u}}{\partial t}+H \boldsymbol{u}\right)+4 \pi G \rho \delta=-\frac{1}{a^{2}} \nabla \cdot[(\boldsymbol{u} \cdot \nabla) \boldsymbol{u}] \\
& \quad+\frac{4}{a^{2}} \nabla \cdot\left\{\varphi\left[(\boldsymbol{u} \cdot \nabla) \boldsymbol{u}-\frac{1}{3}(\nabla \cdot \boldsymbol{u}) \boldsymbol{u}\right]\right\}-\frac{2}{3 a^{2}} \varphi(\boldsymbol{u} \cdot \nabla)(\nabla \cdot \boldsymbol{u}) \\
& \quad-\frac{1}{a^{2}} \Delta\left[(\boldsymbol{u} \cdot \nabla) \Delta^{-1} X_{2}\right]+\frac{1}{a^{2}}(\boldsymbol{u} \cdot \nabla) X_{2}+\frac{2}{3 a^{2}} X_{2}(\nabla \cdot \boldsymbol{u}),
\end{aligned}
$$

where $\varphi$ and $X_{2}$ are the linear and the second-order quantities, respectively, and are defined as

$$
\begin{gathered}
\frac{\Delta}{a^{2}} \varphi=\frac{1}{c^{2}}\left(-4 \pi G \rho \delta+\frac{H}{a} \nabla \cdot \boldsymbol{u}\right) \\
X_{2}=2 \varphi \nabla \cdot \boldsymbol{u}-(\boldsymbol{u} \cdot \nabla) \varphi+\frac{3}{2} \Delta^{-1} \nabla \cdot[\boldsymbol{u} \Delta \varphi+\boldsymbol{u} \cdot \nabla(\nabla \varphi)] .
\end{gathered}
$$

In relativistic perturbation theory, the dimensionless quantity $\varphi$ is proportional to the spatial curvature perturbation in the comoving gauge. All the perturbation variables $\delta, \boldsymbol{u}$, and $\varphi$ can be regarded as equivalently gauge-invariant combinations to nonlinear order. Proper choice of variables and gauge conditions are important to have these equations. Note that the relativistic continuity and Euler equations coincide with those from Newtonian fluid approximation up to the second order in perturbations (Peebles 1980; Noh \& Hwang 2004). Therefore, the perturbative solutions are also the same up to the second order, and pure general relativistic effects appear from the third order. We emphasize that the above equations are valid in the presence of the cosmological constant in the background world model.

An examination of the third-order terms in Equations (4) and (5) shows that the pure third-order terms are simple convolutions of the linear order $\varphi$ with the second-order combinations of fluid variables $\delta$ and $\boldsymbol{u}$. Note that to the linear order, $\varphi$ is a well-known conserved quantity whose amplitude in the growing mode solution is conserved on the super-horizon scales, independent of the changing equation of state or even changing underlying gravitational theories (Hwang \& Noh 2005a). In a flat background without a cosmological constant, the amplitude of $\varphi$ near the horizon scale is directly related to the amplitude of the relative temperature fluctuations of the $\mathrm{CMB}$ as $\delta T / T=\varphi / 5$.

The linear solutions of Equations (4) and (5) are easily found to be

$$
\begin{gathered}
\delta_{1}(\boldsymbol{k}, t)=D(t) \delta_{1}\left(\boldsymbol{k}, t_{0}\right), \\
\theta_{1}(\boldsymbol{k}, t)=-a H D(t) \delta_{1}\left(\boldsymbol{k}, t_{0}\right),
\end{gathered}
$$

where $D(t)$ is the linear growth factor so that $\delta_{1}\left(\boldsymbol{k}, t_{0}\right)$ is the present linear density fluctuation. With these linear solutions, we can perturbatively expand the density contrast $\delta(\boldsymbol{k}, t)=$ $\delta_{1}+\delta_{2}+\delta_{3}+\cdots$, where $\delta_{n}$ is an $n$ th-order quantity in linear density contrast $\delta_{1}\left(\boldsymbol{k}, t_{0}\right)$; we can do the same for $\theta(\boldsymbol{k}, t)$. With this expansion, we can find the full nonlinear solutions of Equations (4) and (5) by using momentum-dependent symmetric kernels as

$$
\begin{aligned}
& \delta(\boldsymbol{k}, t)=\sum_{n=1}^{\infty} D^{n}(t) \int \frac{d^{3} q_{1} \cdots d^{3} q_{n}}{(2 \pi)^{3(n-1)}} \delta^{(3)}\left(\boldsymbol{k}-\sum_{i=1}^{n} \boldsymbol{q}_{i}\right) \\
& \times F_{n}^{(s)}\left(\boldsymbol{q}_{1}, \ldots, \boldsymbol{q}_{n}\right) \delta_{1}\left(\boldsymbol{q}_{1}\right) \cdots \delta_{1}\left(\boldsymbol{q}_{n}\right), \\
& \theta(\boldsymbol{k}, t)=-a H \sum_{n=1}^{\infty} D^{n}(t) \int \frac{d^{3} q_{1} \cdots d^{3} q_{n}}{(2 \pi)^{3(n-1)}} \delta^{(3)}\left(\boldsymbol{k}-\sum_{i=1}^{n} \boldsymbol{q}_{i}\right) \\
& \times G_{n}^{(s)}\left(\boldsymbol{q}_{1}, \ldots, \boldsymbol{q}_{n}\right) \delta_{1}\left(\boldsymbol{q}_{1}\right) \cdots \delta_{1}\left(\boldsymbol{q}_{n}\right) \text {. }
\end{aligned}
$$

Then, Equations (4) and (5) become simple differential equations of $F_{n}^{(s)}$ and $G_{n}^{(s)}$. The general relativistic terms in particular, which explicitly include $k_{H} \equiv a H$, the comoving wavenumber corresponding to the comoving horizon, are reduced to the algebraic equations

$$
\begin{aligned}
& 2 F_{3, \text { Einstein }}-G_{3, \text { Einstein }}=-\frac{5}{2} k_{H}^{2}\left\{2 \frac{\boldsymbol{q}_{1} \cdot \boldsymbol{q}_{3}}{q_{1}^{2} q_{2}^{2}}+\frac{\boldsymbol{q}_{12} \cdot \boldsymbol{q}_{3}}{q_{12}^{2}}\right. \\
& \left.\times\left[-\frac{2}{q_{2}^{2}}+\frac{\boldsymbol{q}_{1} \cdot \boldsymbol{q}_{2}}{q_{1}^{2} q_{2}^{2}}-\frac{3}{2} \frac{\boldsymbol{q}_{12} \cdot \boldsymbol{q}_{1}}{q_{12}^{2} q_{1}^{2}}-\frac{3}{2} \frac{\boldsymbol{q}_{12} \cdot \boldsymbol{q}_{2}}{q_{12}^{2}} \frac{\boldsymbol{q}_{1} \cdot \boldsymbol{q}_{2}}{q_{1}^{2} q_{2}^{2}}\right]\right\},
\end{aligned}
$$




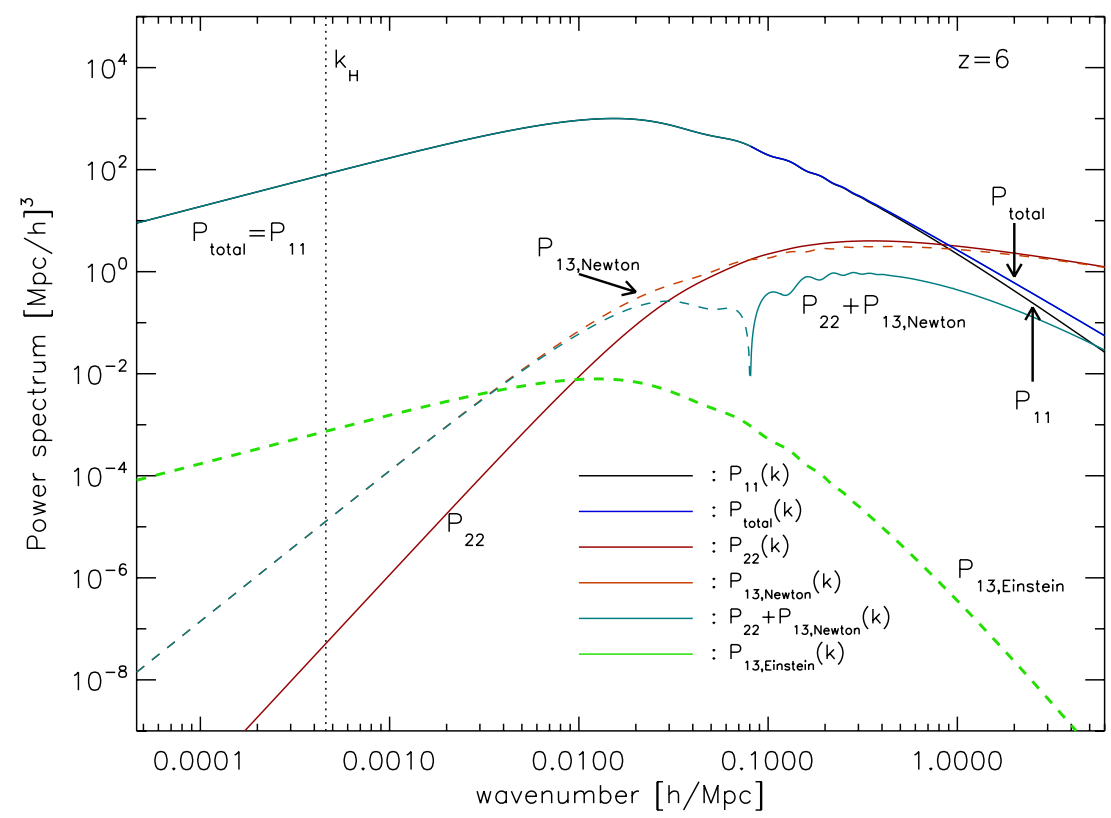

Figure 1. Nonlinear matter power spectrum (solid blue line) and the contribution from each component of Equation $(16)$ at $z=6$. The black, red, and orange lines show the contributions from the Newtonian perturbation theory: $P_{11}, P_{22}$, and $P_{13 \text {, Newton }}$, respectively. The green line shows the general relativistic effect, $P_{13 \text {,Einstein }}(k)$. Note that we take the absolute values for negative terms, and show these with dashed lines: $P_{22}$ and $P_{13}$ are positive and negative, respectively, in all scales. The


negligibly small compared to the linear power spectrum, $P_{11}$ (black).

(A color version of this figure is available in the online journal.)

$$
\begin{aligned}
& \frac{3}{2} F_{3, \text { Einstein }}-\frac{5}{2} G_{3, \text { Einstein }} \\
= & -\frac{5}{2} k_{H}^{2}\left\{\left[\frac{2}{3}+\frac{\boldsymbol{q}_{1} \cdot \boldsymbol{q}_{23}}{q_{1}^{2}}\left(1-\frac{k^{2}}{q_{23}^{2}}\right)\right]\right. \\
& \times\left[-\frac{2}{q_{3}^{2}}+\frac{\boldsymbol{q}_{2} \cdot \boldsymbol{q}_{3}}{q_{2}^{2} q_{3}^{2}}-\frac{3}{2} \frac{\boldsymbol{q}_{23} \cdot \boldsymbol{q}_{2}}{q_{23}^{2} q_{2}^{2}}-\frac{3}{2} \frac{\boldsymbol{q}_{23} \cdot \boldsymbol{q}_{3}}{q_{23}^{2}} \frac{\boldsymbol{q}_{2} \cdot \boldsymbol{q}_{3}}{q_{2}^{2} q_{3}^{2}}\right] \\
& \left.+\frac{1}{q_{3}^{2}}\left[\frac{2}{3} \frac{\boldsymbol{q}_{1} \cdot \boldsymbol{q}_{2}}{q_{2}^{2}}-4\left(\frac{\boldsymbol{q}_{1} \cdot \boldsymbol{q}_{2}}{q_{2}^{2}}-\frac{1}{3}\right) \frac{\boldsymbol{k} \cdot \boldsymbol{q}_{1}}{q_{1}^{2}}\right]\right\},
\end{aligned}
$$

where we have introduced $\boldsymbol{q}_{12 \cdots n}=\sum_{i=1}^{n} \boldsymbol{q}_{i}$. The secondand third-order Newtonian kernels can be found in, e.g., Equations ((2.32), (2.33)) and ((2.34), (2.35)) in Jeong (2010), respectively.

\section{MATTER AND VELOCITY POWER SPECTRA}

From Equation (10), we can find the nonlinear power spectrum, which is defined as

$$
\left\langle\delta\left(\boldsymbol{k}_{1}, t\right) \delta\left(\boldsymbol{k}_{2}, t\right)\right\rangle \equiv(2 \pi)^{3} \delta^{(3)}\left(\boldsymbol{k}_{1}+\boldsymbol{k}_{2}\right) P\left(k_{1}, t\right) .
$$

If we assume perfect Gaussianity for $\delta_{1}$, which is a very good approximation consistent with current observations, any higher order correlation function beyond the linear power spectrum $P_{11}(k)$ can be expressed in terms of it and $P_{11}(k)$ is all that we need to specify the statistics of the density fluctuation $\delta$ : as we will see shortly, all the nonlinear corrections to the power spectrum can be written in terms of $P_{11}$. Then, from Equation (14), we can write, beyond the linear density power spectrum $P_{11}$,

$$
P=P_{11}+P_{22}+P_{13}+\cdots,
$$

with $\left\langle\delta_{i}\left(\boldsymbol{k}_{1}\right) \delta_{j}\left(\boldsymbol{k}_{2}\right)\right\rangle \equiv(2 \pi)^{3} \delta^{(3)}\left(\boldsymbol{k}_{1}+\boldsymbol{k}_{2}\right) s_{i j} P_{i j}\left(k_{1}\right)$. Here, $s_{i j}$ is a symmetric factor which is 1 for $i=j$ and $1 / 2$ otherwise.
The leading nonlinear correction $P_{12}$ includes the bispectrum and thus disappears according to our assumption of Gaussianity for $\delta_{1} . P_{22}+P_{13}$ denotes the next-to-leading order nonlinear correction to the power spectrum. As mentioned above, $P_{13}$ includes general relativistic terms.

The density power spectrum up to the next-to-leading order nonlinear corrections is

$$
\begin{aligned}
P(k, t)= & P_{11}(k, t)+\frac{1}{98} \frac{k^{3}}{(2 \pi)^{2}} \int_{0}^{\infty} d r P_{11}(k r, t) \\
& \times \int_{-1}^{1} d x P_{11}\left(k \sqrt{1+r^{2}-2 r x}, t\right) \\
& \times \frac{\left(3 r+7 x-10 r x^{2}\right)^{2}}{\left(1+r^{2}-2 r x\right)^{2}}+\frac{1}{252} \frac{k^{3}}{(2 \pi)^{2}} P_{11}(k, t) \\
& \times \int_{0}^{\infty} d r P_{11}(k r, t)\left[-42 r^{4}+100 r^{2}-158\right. \\
& \left.+\frac{12}{r^{2}}+\frac{3}{r^{3}}\left(r^{2}-1\right)^{3}\left(7 r^{2}+2\right) \log \left|\frac{1+r}{1-r}\right|\right] \\
& +\frac{5}{56}\left(\frac{k_{H}}{k}\right)^{2} \frac{k^{3}}{(2 \pi)^{2}} P_{11}(k, t) \int_{0}^{\infty} d r P_{11}(k r, t) \\
& \times\left[86 r^{2}-130-\frac{72}{r^{2}}+\frac{1}{r^{3}}\left(36+53 r^{2}-46 r^{4}\right.\right. \\
& \left.\left.-43 r^{6}\right) \log \left|\frac{1+r}{1-r}\right|\right] \\
\equiv & P_{11}+P_{22}+P_{13, \text { Newton }}+P_{13, \text { Einstein }},
\end{aligned}
$$

where $r$ and $x$ are the magnitude of dummy integration momentum $\boldsymbol{q}$ and the cosine between $\boldsymbol{q}$ and $\boldsymbol{k}$, respectively, introduced as $q \equiv r k(0 \leqslant r \leqslant \infty)$ and $\boldsymbol{k} \cdot \boldsymbol{q} \equiv k^{2} r x(-1 \leqslant x \leqslant 1)$. We have divided $P_{13}$ into the Newtonian part $P_{13 \text {,Newton }}$ and the general relativistic contribution $P_{13 \text {,Einstein }}$. Compared with $P_{13 \text {,Newton }}$, the general relativistic contribution $P_{13 \text {,Einstein }}$ is multiplied by 


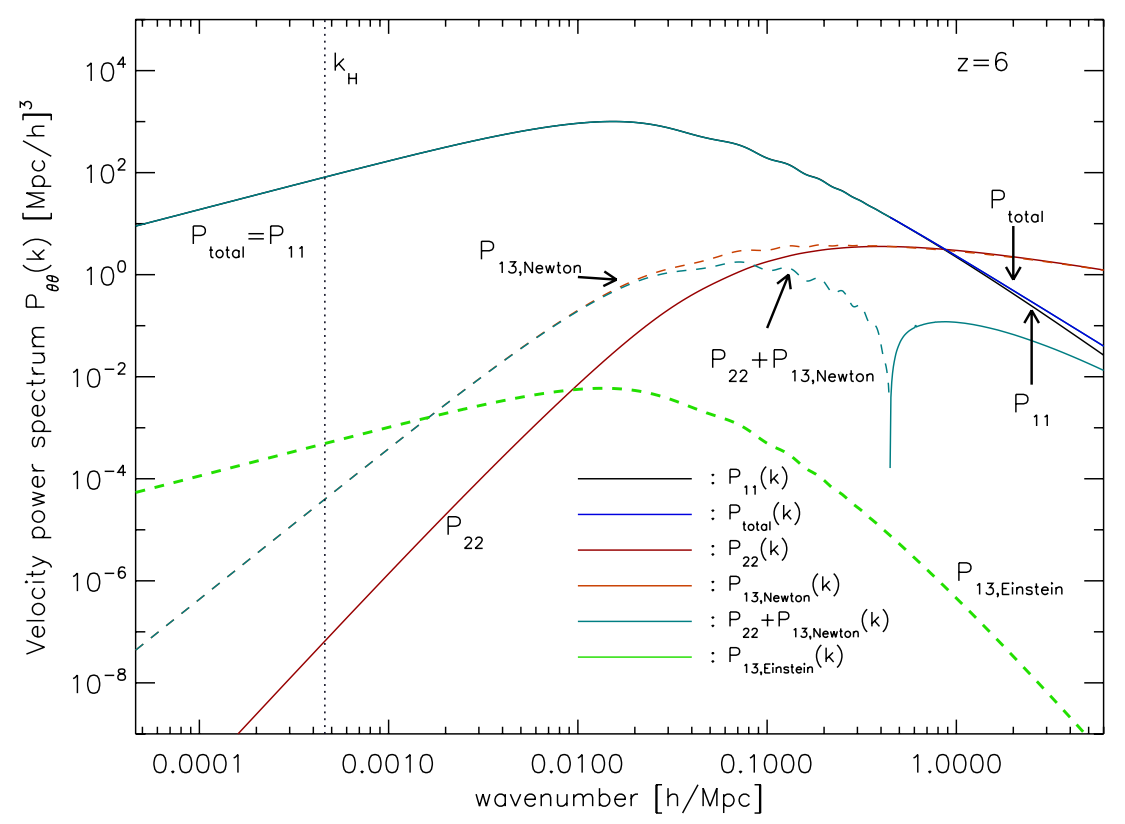

Figure 2. Same as Figure 1, but for the velocity power spectrum $P_{\theta \theta}(k)$.

(A color version of this figure is available in the online journal.)

a factor $\left(k_{H} / k\right)^{2}$, where $k_{H} / k$ is the ratio between a scale of interest and the horizon scale, and is thus highly suppressed far inside the horizon.

In Figure 1, we present the total power spectrum of Equation (16), along with its components $P_{11}, P_{22}, P_{13 \text {, Newton, }}$

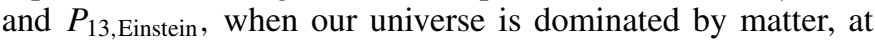
$z=6$. The linear power spectrum is calculated by the CAMB (Lewis et al. 2000) code with the maximum likelihood cosmological parameters given in Table 1 of Komatsu et al. (2009; "WMAP+BAO+SN"). Figure 1 shows that the general relativistic contribution $P_{13 \text {,Einstein }}$ is smaller than the linear power spectrum $P_{11}$ on all cosmological scales.

Let us examine $P_{13 \text {,Einstein }}$ more closely. For notational simplicity, we shall abbreviate the integration in $P_{13 \text {, Einstein as }}$ $\int d r P_{11}(k r, t) f(r)$. Then, the scale dependence of $P_{13 \text {,Einstein }}$ can be understood as follows. First, setting $k r=q$, we find that $P_{13 \text {,Einstein }} \sim P_{11}(k) f(q / k)$. On small scales $(k \gg$ $\left.0.01 \mathrm{~h} \mathrm{Mpc}^{-1}\right), q / k$ is also small, and by using Taylor expansion of $f(r)=-(656 / 15) r^{2}+\mathcal{O}\left(r^{4}\right)$ we find $P_{13 \text {,Einstein }} \sim k^{-2} P_{11}$. On the other hand, in large scale limit $\left(k \ll 0.01 h \mathrm{Mpc}^{-1}\right)$ where $q / k$ takes larger value, $f(r)=-752 / 3+\mathcal{O}\left(r^{-2}\right)$ and $P_{13 \text {, Einstein }}$ has a scale dependence of $P_{13 \text {, Einstein }} \sim P_{11}$. Numerical calculation reveals that $P_{13 \text {,Einstein }}$ is smaller than $P_{11}$ by a factor of $10^{-5}$ on large scales. Our result shows that the leading order nonlinear power spectrum is finite in both infrared and ultraviolet regions. $^{6}$

We can proceed in the same way to compute the power spectrum of the peculiar velocity. As in Equation (14), we can define

$$
\left\langle\theta\left(\boldsymbol{k}_{1}, t\right) \theta\left(\boldsymbol{k}_{2}, t\right)\right\rangle \equiv(2 \pi)^{3} \delta^{(3)}\left(\boldsymbol{k}_{1}+\boldsymbol{k}_{2}\right) P_{\theta \theta}\left(k_{1}, t\right),
$$

\footnotetext{
6 The previous result reporting infrared divergence in $P_{13 \text {,Einstein }}$ (Noh et al. 2009) turns out to be due to an incorrect calculation of the power spectrum: the

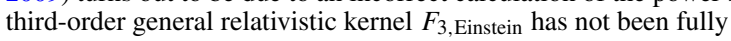
symmetrized, thus causing logarithmic infrared divergence in $P_{13 \text {,Einstein. }}$
}

and we can find

$$
\begin{aligned}
k_{H}^{-2} P_{\theta \theta}(k, t)= & P_{11}(k, t)+\frac{1}{98} \frac{k^{3}}{(2 \pi)^{2}} \int_{0}^{\infty} d r P_{11}(k r, t) \\
& \times \int_{-1}^{1} d x P_{11}\left(k \sqrt{1+r^{2}-2 r x}, t\right) \\
& \times \frac{\left(r-7 x+6 r x^{2}\right)^{2}}{\left(1+r^{2}-2 r x\right)^{2}} \\
& +\frac{1}{84} \frac{k^{3}}{(2 \pi)^{2}} P_{11}(k, t) \int_{0}^{\infty} d r P_{11}(k r, t) \\
& \times\left[-6 r^{4}+4 r^{2}-82+\frac{12}{r^{2}}+\frac{3}{r^{3}}\left(r^{2}-1\right)^{3}\right. \\
& \left.\times\left(r^{2}+2\right) \log \left|\frac{1+r}{1-r}\right|\right] \\
& +\frac{5}{56}\left(\frac{k_{H}}{k}\right)^{2} \frac{k^{3}}{(2 \pi)^{2}} \int_{0}^{\infty} d r P_{11}(k r, t) \\
& \times\left[46 r^{2}-50-\frac{144}{r^{2}}+\frac{1}{r^{3}}\right. \\
& \left.\times\left(-23 r^{6}-50 r^{4}+r^{2}+72\right) \log \left|\frac{1+r}{1-r}\right|\right] .
\end{aligned}
$$

Figure 2 shows the nonlinear velocity power spectrum of Equation (18) for exactly the same cosmology as Figure 1. As in the case of the total matter power spectrum, the nonlinear general relativistic correction is negligibly small for all scales. It is because the third-order kernel for velocity $G_{3 \text {,Einstein be- }}$ haves in the same way as that for the matter density $F_{4 \text {,Einstein }}$ in both the large $(r \rightarrow 0)$ and small $(r \rightarrow \infty)$ scale limits: $\lim _{r \rightarrow 0} g(r)=-(368 / 15) r^{2}+\mathcal{O}\left(r^{4}\right)$ and $\lim _{r \rightarrow \infty} g(r)=$ $-496 / 3+\mathcal{O}\left(r^{-2}\right)$ when denoting the last integration in Equation (18) as $\int d r P_{11}(k r) g(r)$. 


\section{CONCLUSION}

To conclude, in this paper we have examined the general relativistic nonlinear contributions to the density and velocity power spectra. We were pleasantly surprised to find that the purely general relativistic effects are completely negligible on all cosmologically relevant scales, even outside the horizon. It is interesting to see that the linear power spectrum totally dominates even outside the horizon. Our conclusion has the following important implication. As the general relativistic effect is very small, Newtonian theory can be safely applied to the nonlinear evolution of cosmic structure on all cosmologically relevant scales. In the literature, it has been common to use Newtonian gravity to study the nonlinear clustering properties of LSS without justifying that approach. The result we present in this paper provides a confirmation of using Newtonian gravity to handle nonlinear clustering in cosmology.

J.G. thanks Misao Sasaki and Takahiro Tanaka for useful conversations, and is grateful to the Yukawa Institute for Theoretical Physics, Kyoto University for hospitality during the workshop YITP-W-10-10 where part of this work was carried out. This work was supported in part by a Robinson prize postdoctoral fellowship at the California Institute of Technology (D.J.), a VIDI and a VICI Innovative Research Incentive Grant from the Netherlands Organisation for Scientific Research (NWO; J.G.), the Mid-career Research Program through National Research
Foundation funded by the MEST (No. 2010-0000302; H.N.), and the Korea Research Foundation Grant funded by the Korean Government (KRF-2008-341-C00022; J.H.).

\section{REFERENCES}

Arnowitt, R., Deser, S., \& Misner, C. W. 2008, Gen. Rel. Grav., 40, 1997 Bardeen, J. M. 1980, Phys. Rev. D, 22, 1882

Bardeen, J. M. 1988, in Cosmology and Particle Physics, ed. L.-Z. Fang \& A. Zee (London: Gordon \& Breach), 1

Bernardeau, F., Colombi, S., Gaztañaga, E., \& Scoccimarro, R. 2002, Phys. Rep., 367,1

Fry, J. N. 1994, ApJ, 421, 21

Goroff, M. H., Grinstein, B., Rey, S., \& Wise, M. B. 1986, ApJ, 311, 6

Hwang, J., \& Noh, H. 2005a, Phys. Rev. D, 71, 063536

Hwang, J., \& Noh, H. 2005b, Phys. Rev. D, 72, 044012

Jeong, D. 2010, PhD thesis, Univ. Texas at Austin (http://hdl.handle.net/ 2152/ETD-UT-2010-08-1781)

Jeong, D., \& Komatsu, E. 2006, ApJ, 651, 619

Jeong, D., \& Komatsu, E. 2009, ApJ, 691, 569

Kim, J., Park, C., Gott, J. R., \& Dubinski, J. 2009, ApJ, 701, 1547

Komatsu, E., et al. 2009, ApJS, 180, 330

Komatsu, E., et al. 2010, ApJS, in press (arXiv:1001.4538)

Lewis, A., Challinor, A., \& Lasenby, A. 2000, ApJ, 538, 473

Makino, N., Sasaki, M., \& Suto, Y. 1992, Phys. Rev. D, 46, 585

Noh, H., \& Hwang, J. 2004, Phys. Rev. D, 69, 104011

Noh, H., \& Hwang, J. 2008, Phys. Rev. D, 77, 123533

Noh, H., Jeong, D., \& Hwang, J. 2009, Phys. Rev. Lett., 103, 021301

Peebles, P. J. E. 1980, The Large-scale Structure of the Universe (Princeton, NJ: Princeton Univ. Press)

Smoot, G. F., et al. 1992, ApJ, 396, L1

Vishniac, E. T. 1983, MNRAS, 203, 345 